\title{
Technical Overview and First Results of the Half-Size ITER NNBI Source
}

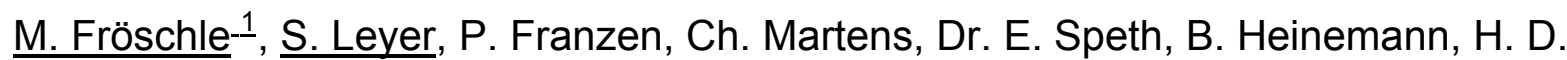
Falter, U. Fantz, W. Kraus, R. Riedl,

\begin{abstract}
IPP Garching is currently developing a radio frequency (RF) driven ion source for the ITER negative ion neutral beam injection (NNBI) as an alternative to the presently foreseen filament source. The RF source has demonstrated the ITER requirements concerning negative ion current and electron/ion ratio at the required source pressure for small pulse length and small extraction area. The next goals are the extension to long pulses and large area sources at two further test facilities. The negative ion source at RADI - the test bed is located at the place of the former Wendelstein 7 AS RADial Injector and uses some of its components - has roughly half the size of the ITER source and is therefore called the Half-Size Source. It was recently commissioned at the IPP Garching. RADI is devoted to demonstrate the required plasma homogeneity of a large RF driven source, to test an ITER relevant RF circuit and to show the scalability of the IPP RF source. Having no large area extraction the source performance will be demonstrated with an extensive diagnostic and modeling program. This paper will present the results of the first plasma discharges and describe the main technical features of RADI.
\end{abstract}

\section{Introduction}

The plasma heating and current drive NNBI system for ITER requires a $\mathrm{D}^{-}$ion current density of $200 \mathrm{~A} / \mathrm{m}^{2}$ within a pulse length of up to one hour, for a source that will be $1.5 \times 0.6 \mathrm{~m}^{2}$ in size [1][2]. The electron/ion ratio must not exceed 1 and the source filling pressure has to be $0.3 \mathrm{~Pa}$ or lower, in order to reduce the power loading of the grids and the stripping losses - the untimely deionisation of the negative ions by loosing their additional electron in the acceleration system.

The development of the source was initially concentrated on filament arc sources as described in the ITER reference design [1][2]. As an interesting alternative, IPP Garching is currently developing RF driven negative hydrogen ion sources [3]. RF sources offer substantial advantages in particular their maintenance free operation in contrast to the arc sources having a limited filament lifetime.

\footnotetext{
${ }^{1}$ Corresponding Author: Max-Planck-Institut für Plasmaphysik

Boltzmannstr. 2

85748 Garching, Germany

E-mail: markus.froeschle@ipp.mpg.de

Phone: +49 8932992621

Fax: +49 8932992558
} 
The ITER requirements concerning ion current density, electron/ion ratio and source filling pressure have already been achieved or even exceeded at the IPP test bed BATMAN (Bavarian Test Machine for Negative ions) for small extraction area $\left(\sim 70 \mathrm{~cm}^{2}\right)$ and limited pulse length $(<4 \mathrm{~s})$ [3][4]. The further development concentrates now on long pulse operation at the test bed MANITU (Multi Ampere Negative Ion Test Unit) [5] and on large extraction areas at the test facility RADI with its so-called Half-Size Source (inner height $0.80 \mathrm{~m}$, inner width $0.76 \mathrm{~m}$ ) [6]. RADI uses the tank of the former Wendelstein 7 AS RADIal injector.

This test facility was built in order to demonstrate the required homogeneity of large RF plasmas. As an intermediate step between the small source at the test bed BATMAN and the full-size ITER source, the Half-Size Source will also show the scalability of the IPP RF source. The source is further devoted to test the geometry and number of drivers as well as an ITER-like RF circuit. Full size extraction is presently not foreseen but included in a future plan.

RADI will be equipped with a Molybdenum dummy grid, matching the conductance of the ITER grid system and hence the gas flow conditions of the ITER source. In order to get some information about possible ion and electron currents, local single aperture extraction with a Faraday cup system (see further in paragraph 4) is planned [6].

An extensive diagnostic and modeling program is accompanying those activities. The main parameters determining the performance of the source are the negative ion and electron density in front of the grid as well as the homogeneity of their profiles across the grid. Those are measured by optical emission spectroscopy [7][8][9][10], Langmuir probes [11], laser detachment [11] and cavity ring down [12][13].

This paper will give an overview of the setup of RADI and presents the first plasma discharges.

\section{Principle Design of the IPP RF Driven Negative Ion Source}

Figure 1 shows the principle design of the IPP RF negative ion source [14][15].

The RF coil, which is connected via a matching unit to the RF generator, is watercooled and in the present design wound in 6 turns over the discharge vessel. This consists of a alumina or quartz tube and is mounted onto the back of the source body. Inside a tungsten-coated copper Faraday screen protects the ceramics against the plasma. The Faraday screen has slits in axial direction (can be seen in figure 8 as well) and is therefore permeable for the RF, so that an inductive coupling to the plasma is possible.

It is well known, that in a caesiated source the amount of extractable negative ions produced by the surface process exceeds the one produced by the volume process by far [3]. Caesium reduces the work-function of the surface which leads to a strong increase of the negative ion yield [3]. Hence Caesium is released from a Caesium oven into the source. It covers the source surfaces including the plasma grid surface, where the extracted ions are produced. 


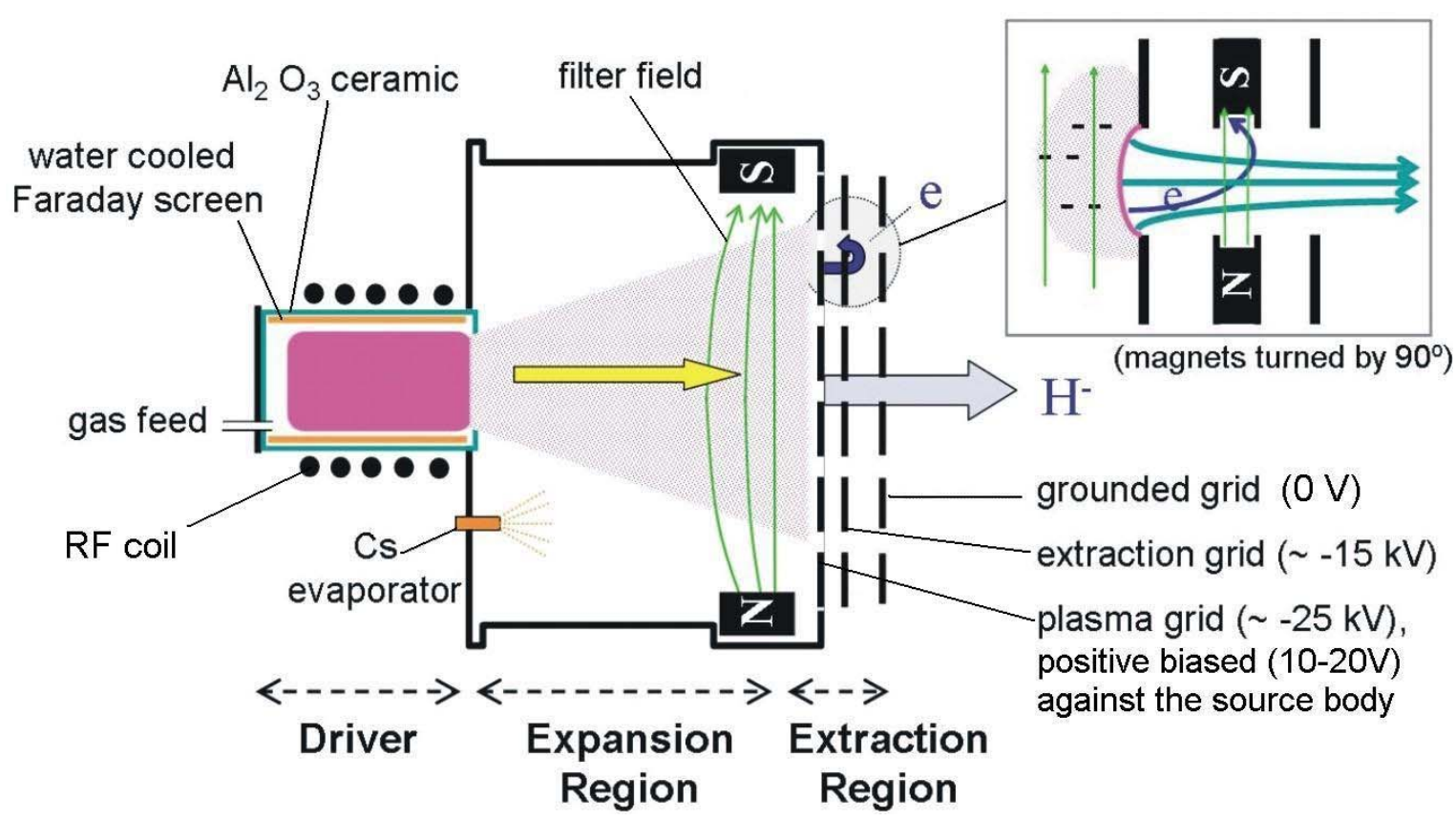

Figure 1: Schematic view of the IPP RF source. The extraction region is shown in detail; the magnets in the extraction grid are shown rotated by $90^{\circ}$.

A certain control of the Caesium coverage of the plasma grid can be obtained by the Caesium evaporation rate of the oven and the temperature of the source body and the plasma grid [3]. Best performance with the IPP negative ion sources was achieved when the plasma grid temperature was set between 100 and $200^{\circ} \mathrm{C}$, while the source body surface was set to about $40^{\circ} \mathrm{C}$ [3].

In a magnetic filter field the electron density is decreased and the hot electrons are cooled down to temperatures below $2 \mathrm{eV}$. This lowers the rate of co-extracted electrons and the destruction of negative ions by collisions with electrons.

Negative ions which are produced at the plasma grid surface can be extracted, if they reach the meniscus shaped shell slightly above the extraction holes of the plasma grid, where the potential of the extraction electrode extends into the plasma.

The co-extracted electrons are deflected by magnets, located in the extraction grid (figure 1, top right, where the magnets are drawn turned by $90^{\circ}$ ). It is assumed, that the electron deflection magnets in the extraction electrode have an important influence as electron filter also. Up to now, best results have been achieved with a filter field and an electron deflection field which are perpendicular to one another. 


\section{Experimental Setup}

The RADI test facility is realized as a very flexible system with:

$>$ variable source depth,

$>$ variable filter fields (PG-Filter, permanent magnets),

$>$ variable confinement field,

$>$ variable geometry and number of drivers, and

$>$ the possibility to run the drivers in air or in vacuum.

A detailed description can be found in [6].

The setup of RADI is shown in figure 2 and figure 3 .

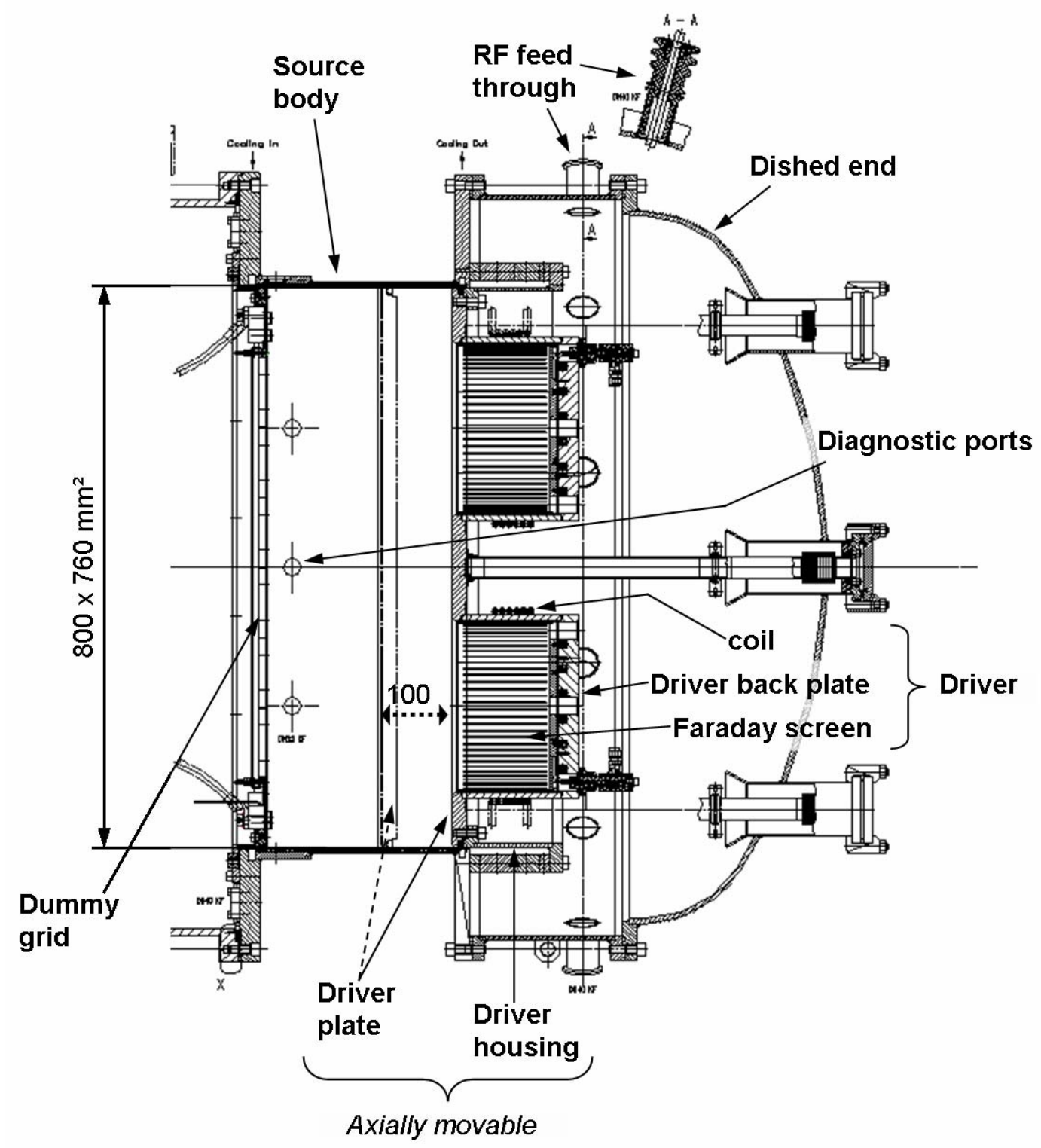

Figure 2: Vertical cross section of the Half-Size Source, here with the optional dished end to run the drivers in vacuum. The depth of the source can be varied in the range of $100 \mathrm{~mm}$ by moving the driver housing with driver plate and drivers. 


\section{Source Body}

The Half-Size Source houses four drivers to create the plasma. These drivers are fixed by four bolts each on the "driver plate" which is connected with the "driver housing", see figure 3 . After venting, the driver housing can be moved axially into the source body to allow the test of different depths $(160 \ldots 260 \mathrm{~mm})$ of the expansion region. An axially pressed $10 \mathrm{~mm}$ O-ring is sealing laterally onto the inner wall of the source body.

The source body consists of a front and a rear flange and the side walls. Near the grid system the side walls are $15 \mathrm{~mm}$ thick and 16 diagnostic ports -3 in horizontal and 5 in vertical direction - are integrated there. The diagnostic ports have a clearance of $40 \mathrm{~mm}$, which permits also an axial resolution of the lines of sight of the optical emission spectroscopy, located directly above the grid. The rest of the side walls are only $6 \mathrm{~mm}$ thick, to allow attachment of confinement and filter magnets outside onto the walls.

The side walls are equipped with cooling channels, for example the $6 \mathrm{~mm}$ thin side walls are deep drilled with 128 boreholes of $3 \mathrm{~mm} \varnothing$ and $230 \mathrm{~mm}$ length. To assure a uniform temperature distribution, the inner side of the source body is electro-plated with copper.

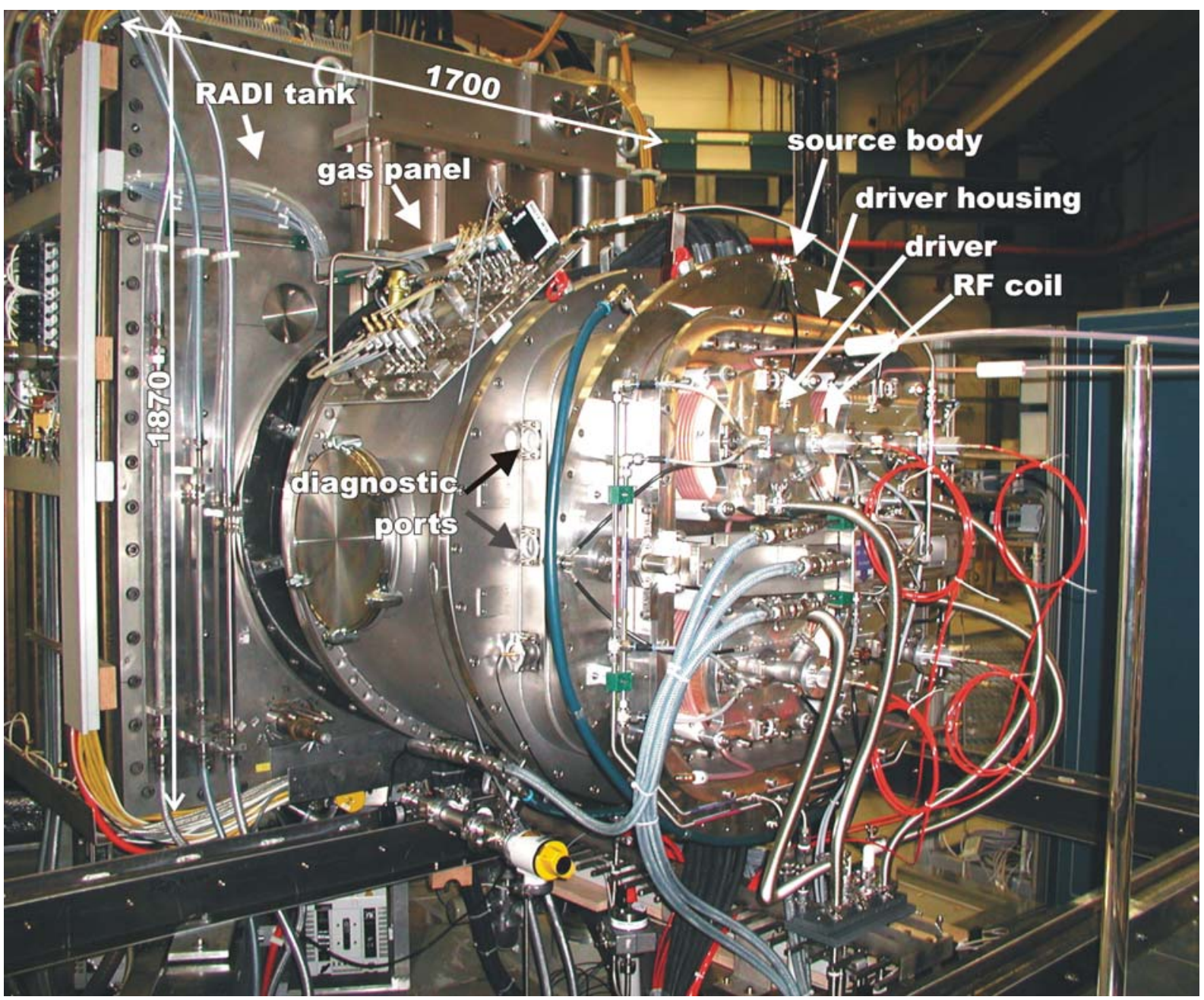

Figure 3: Photograph of the RADI test bed 


\section{Dummy Grid and Filter Field}

A "Dummy Grid" made of Molybdenum simulates the conductance and the gas flow of the ITER extraction system (approx.: $12 \mathrm{~m}^{3} / \mathrm{s}$ ). The conductance is adjustable by movable chevrons (figure 4), which are located behind the slits of the grid.

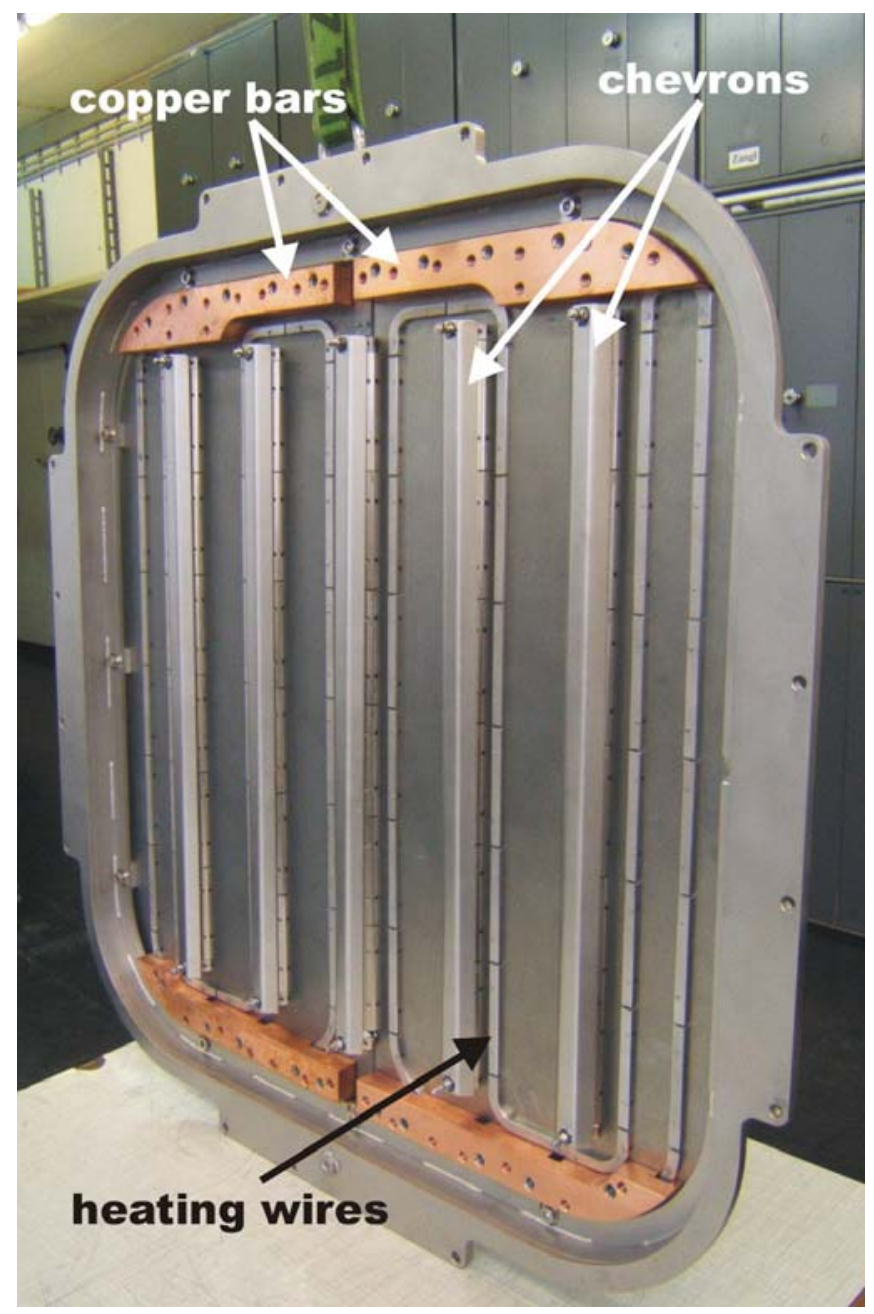

Figure 4: Dummy Grid shown from the rear side. The five "chevrons" which cover the slits can be moved in order to vary the gas conductance of the grid. The PG current is fed through the copper bus bars.

The grid is equipped with heating wires and thermocouples, to provide a temperature control of up to $200^{\circ} \mathrm{C}$.

The magnetic filter field at RADI can be produced either by a strong current flowing vertically through the grid, the so called PG-Filter (where "PG" stands for plasma grid), which is also foreseen for the ITER source. The system is equipped with a $15 \mathrm{~V}$, 5kA power supply, which produces a magnetic flux density of about $10 \mathrm{mT}$ in front of the grid. Another option is to attach permanent magnets to the source from outside, as done at the smaller IPP sources BATMAN and MANITU. The third possibility is to insert vertical "rods" into the source, namely between the intended extraction areas (grid segments) of the ITER source, where diagnostic

ports are foreseen at RADI. The rods are cooled and either bear permanent magnets or a coil.

All these variants create a horizontal magnetic field. Their different distributions of the magnetic field strength are compared in figure 5. The upper part of the figure shows their distributions in horizontal direction $1 \mathrm{~cm}$ in front of the grid. It can be seen, that the field of the "magnets outside" decreases rapidly from the source sidewalls to the centre. Therefore the permanent magnets attached from outside are not expected to be the exclusive solution for large sources, but they can be useful to form the shape of the total field. The rods in this figure have to be imagined between the grid segments, which are indicated as thick bars. The field strength of the rod magnets runs out of the graphic at the place of themselves. The lower part of the figure explains the distribution in axial direction between grid and driver. It can be seen, that the magnetic field of the PG current reaches much further backwards into the source as the rod filter. 

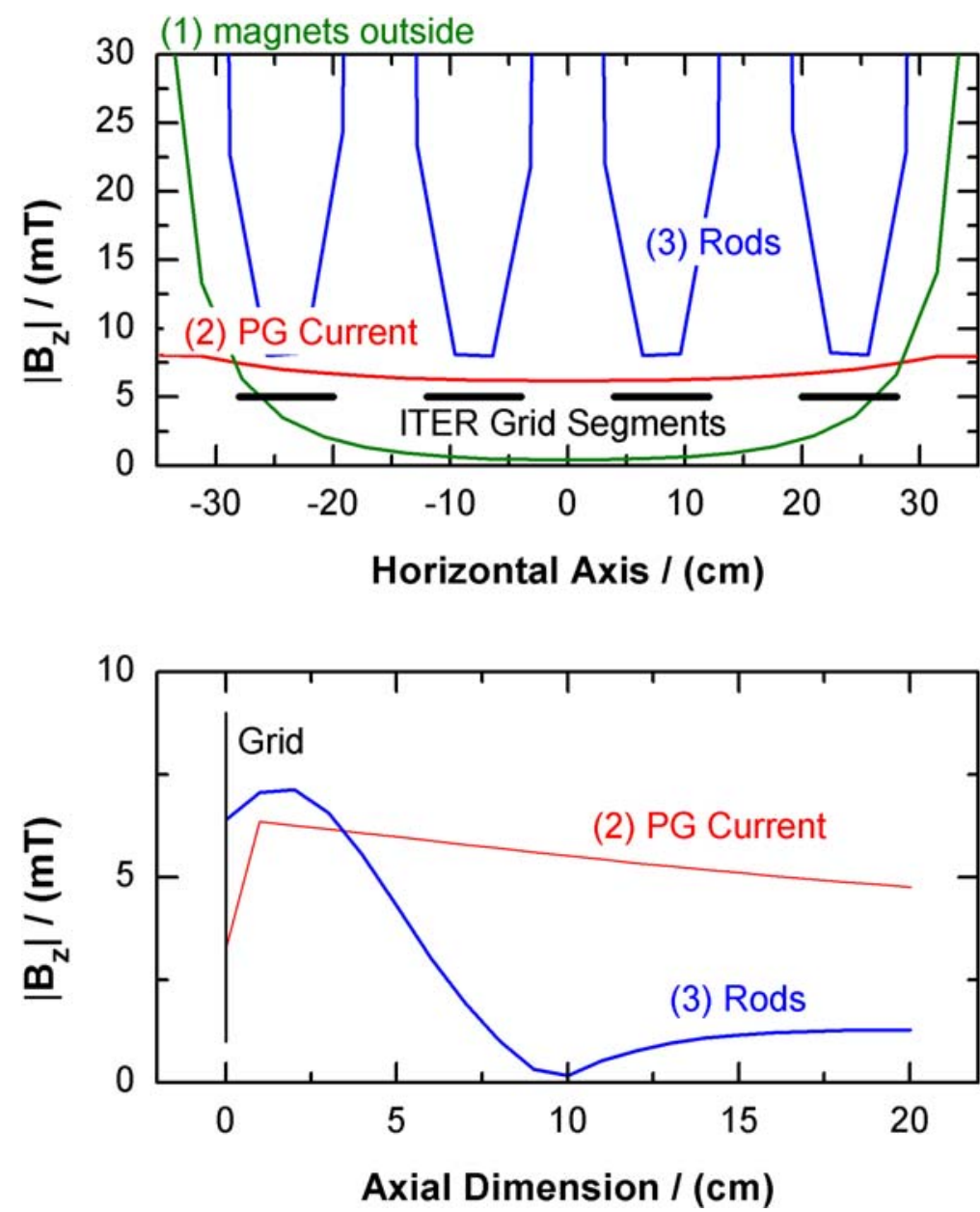

Figure 5: Calculated magnetic filter field by (1) permanent magnets outside the source, (2) by a plasma grid current of 10 $k A$, and (3) by magnet rods inserted in the source with the magnets being located $1 \mathrm{~cm}$ above the grid. Top: Horizontal profiles $1 \mathrm{~cm}$ in front of the grid. The grid segments (extraction areas) are indicated by the thick black lines. Bottom: Axial profiles into the source at the centre of one grid segment. As the absolute values are shown, the curve of the rod filter starts rising again at $10 \mathrm{~cm}$ where the magnetic field reverses.

\section{The RF Circuit}

For RADI two $1 \mathrm{MHz}$ generators are available. They are rated for $180 \mathrm{~kW}$ each and for a pulse length of $30 \mathrm{~s}$. Their typical electrical efficiency is $60 \%$. In the actual setup with four drivers, one RF generator supplies via a transmission line and a matching unit near the source the coils of the horizontal pair of drivers. The advantage of this configuration, which is also intended for ITER, is that the RF power of the generators can be set individually, so that plasma non-uniformities in the vertical, longer dimension, which were observed in experiments with large filament sources [16][17], can be compensated.

In order to couple the RF power to the plasma the impedance of the plasma has to be transformed to the $50 \Omega$ output impedance of the generator by a matching unit. The plasma resistance in one driver is in the range of $2-3 \Omega$ depending on the plasma parameters. The RF circuit is shown in figure 6. 


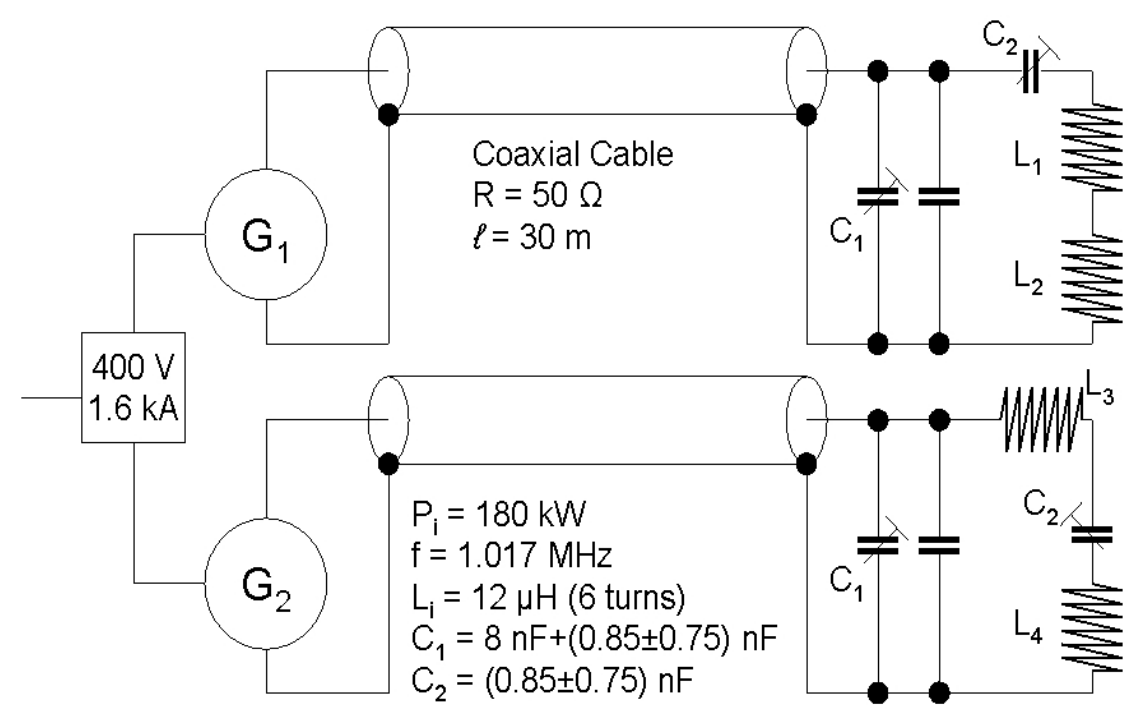

Figure 6: RF circuit for RADI with the 4 driver configuration (L1 - L4). The matching unit near the source is drawn in two possible arrangements to be tested ('CLL' or ' $L C L$ ', respectively); during operation only one arrangement for both circuits will be used.

The matching circuit consists of two capacitors, one parallel (C1) and one in series (C2) to the two coils (drivers). The matching is done by changing the motor driven capacitors ( $\mathrm{C} 1$ is here realised by a fixed and an adjustable capacitor). The matching box with the capacitors is shown in figure 7. As for the ITER RF circuit variable capacitors near the source are hardly feasible, a different concept is planned. There the oscillating circuit at the source contains only a fixed capacity and the matching will be done by the variable frequency of the RF generator.

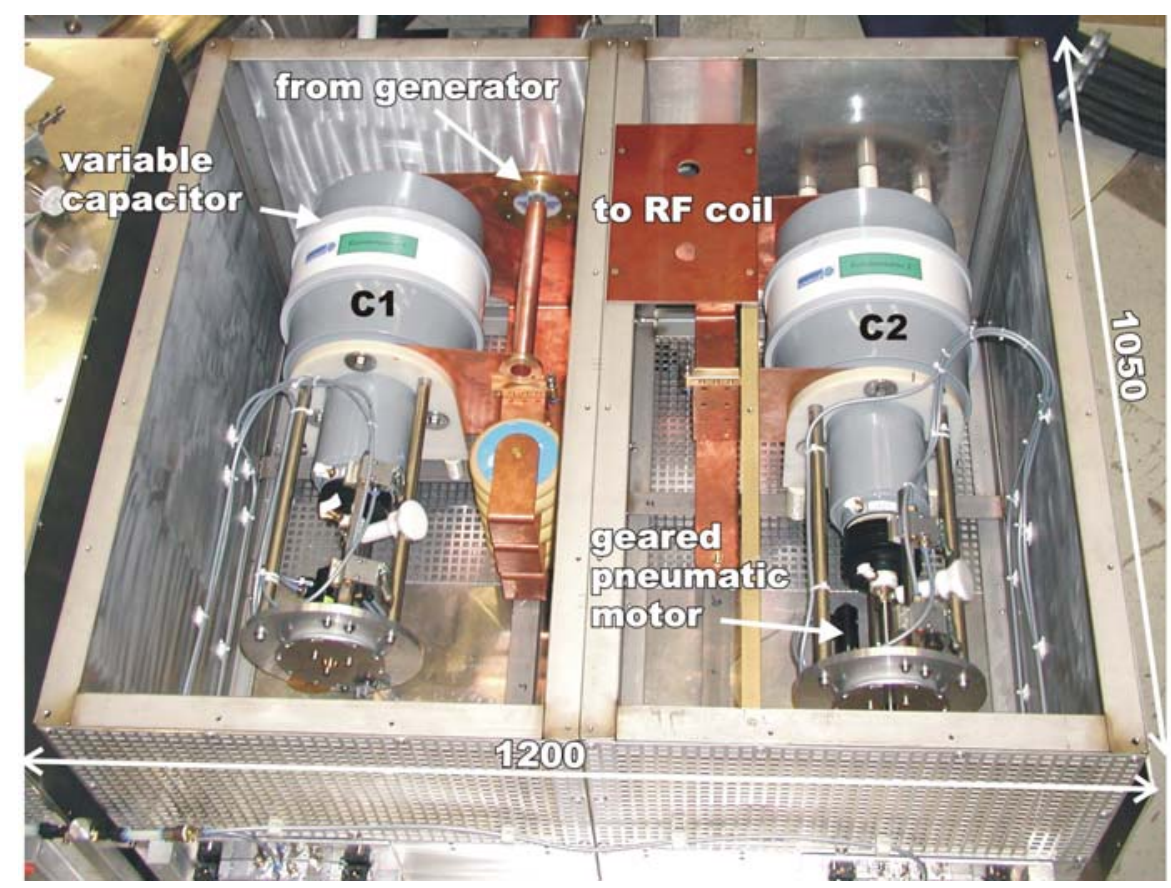

Figure 7: One of the two matching units of RADI. Each unit consists of two variable capacitors (C1 and $\mathrm{C2}$ ) which are driven by a geared pneumatic motor. 
The experience with RADI will influence the ITER design concerning for example cooling requirements for $\mathrm{cw}$ operation, optimizing the number of coil turns and coil insulation and the arrangement of the oscillating circuit ('CLL' or 'LCL', see figure 6).

\section{Peripheral Equipment}

The vacuum system of RADI consists of a turbo molecular pump and 3 titanium getter pumps with a total pumping speed of $160,000 \mathrm{l} / \mathrm{s}$. The tank of the former radial injector of Wendelstein 7-AS with a volume of $5 \mathrm{~m}^{3}$ is used for the vacuum vessel.

The gas supply of the source consists of two parallel systems, one for Hydrogen or Deuterium, the other for inert gases. Near the drivers an array of pneumatic valves is installed, each of them has a specific, calibrated flow rate. The total flow is regulated by the combination of the open valves and is measured in addition by a flow meter. Next to the drivers the piping branches out into four equivalent hoses that lead into the driver back plate to feed the source. The equality of these hoses is important to ensure an equal gas flow and plasma impedance in all drivers.

The Caesium supply is performed by two Caesium ovens. Each oven is equipped with three Caesium ampoules, each containing $1 \mathrm{~g}$ of Caesium. The ampoules are inside a vacuum cover of thin metal, where the glass ampoule can be broken from outside without braking the source vacuum or exposing Caesium to the air. The amount of Caesium evaporated into the source is controlled by the temperature of the oven. As Caesium condenses easily at cold surfaces, the temperature control of the oven has control loops of thermo couples and heating wires to avoid cold spots and Caesium sinks in the piping of the oven.

For the same reason - to avoid Caesium sinks - all inner surfaces of the source have to be temperature controlled, which is managed by the temperature control of the cooling water.

\section{Source Diagnostics}

The performance of the Half-Size Source is measured by the plasma parameters in front of the grid. Those are mainly the negative ion and electron density as well as the Caesium neutral and ion density across the grid. Hence RADI has several diagnostic tools - figure 8 shows the diagnostic ports from an inside view - like optical emission spectroscopy, Langmuir probes, laser detachment and cavity ring down spectroscopy, whose functional capability under RF conditions was shown at the other IPP test beds. The diagnostics are calibrated to the extracted current densities at BATMAN. A $D^{-}$density of $1 \times 10^{17} \mathrm{~m}^{-3}$ in front of the grid $(\sim 3 \mathrm{~cm})$ is determined for an extracted current density of $22 \mathrm{~mA} / \mathrm{cm}^{2}$ [6], in accordance with theoretical estimations [18][19].

In the following the different diagnostic tools are described.

\section{Optical Emission Spectroscopy}

Optical emission spectroscopy [6][8][9] is a non-invasive diagnostic tool for negative ion sources. RADI has a multitude of diagnostic ports parallel and perpendicular to 
the plasma grid, so that spatial resolution can be achieved for example to resolve contributions from the individual drivers. Time resolved measurements allow monitoring of the Caesium and thus the study of the Caesium dynamics in the source. The new technique of measuring the negative ion density via the $\mathrm{H} \alpha / \mathrm{H} \beta$ ratio [10] - developed at IPP — provides an in situ monitoring of the $\mathrm{H}^{-}$density close to the grid. Determination of local quantities can be done via tomography.

RADI will be initially equipped with two three channel survey spectrometers $\left(\Delta \lambda_{F W H M} \approx 1-1.8 \mathrm{~nm}, \lambda=200-870 \mathrm{~nm}, 100 \mathrm{~ms}\right.$ time resolution), recording the plasma parameters of 6 lines of sight simultaneously.

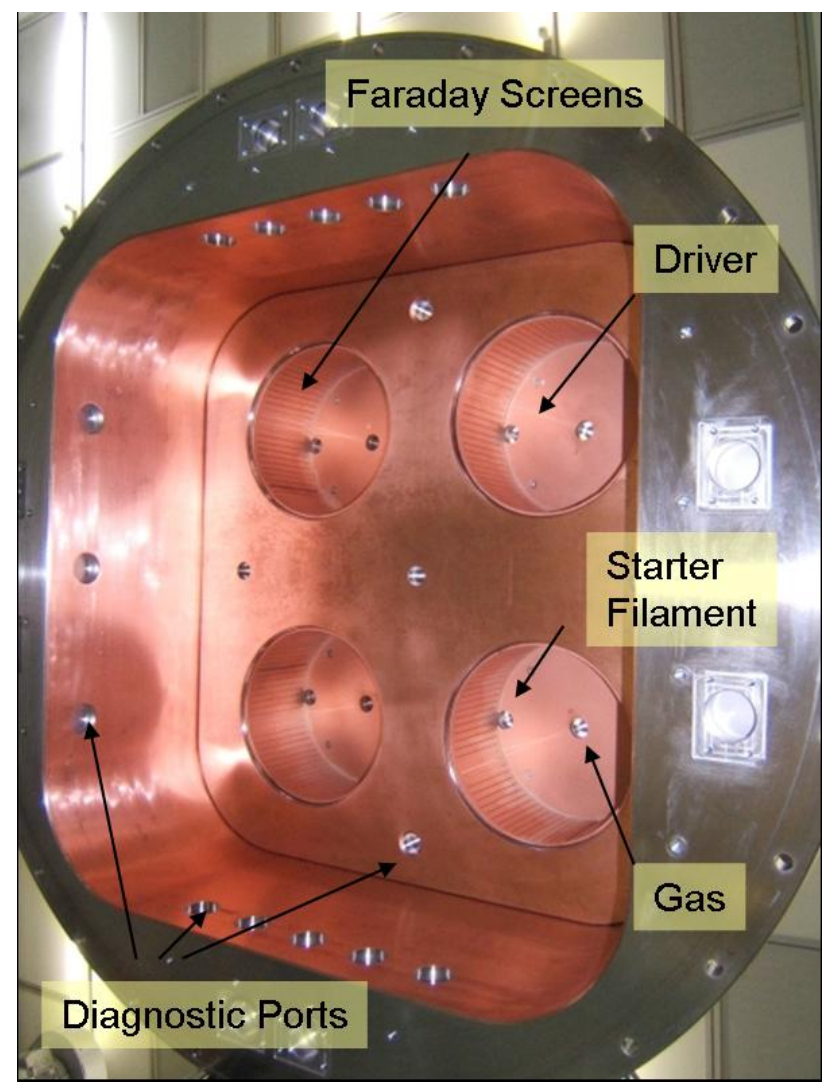

Figure 8: Picture of the inside of the HalfSize Source on RADI. The drivers with the Faraday screens, the ports for the gas inflow, the starter filaments and the diagnostics are shown.

\section{Langmuir Probes}

Two moveable Langmuir [11] probes RADI will be equipped with are a copy of the SMARTPROBE ${ }^{\text {TM }}$ Langmuir probe, which was adapted at the RF source at BATMAN in collaboration with the Charkov University, Ukraine ${ }^{2}$. Spatial profiles can be obtained by a motor-driven positioning system with a maximum distance of $40 \mathrm{~cm}$ from the source edge.

Langmuir probes are known to be very sensitive in the presence of magnetic fields [11]. Although the applied fields are low, their influence can be checked in RADI, since the magnetic filter field can be varied and switched off easily.

The results of the probe, which are mainly the plasma potential and the electron density, provide input data in the evaluation of spectroscopic data and for plasma modelling.

\footnotetext{
${ }^{2}$ Contact: Prof. V. Dudin, University of Charkov, Ukraine
} 


\section{Cavity Ring Down Spectroscopy (CRDS)}

Cavity ring down spectroscopy [12][13] is a diagnostic method based on the absorption technique, namely the absorption of the laser light due to the photo detachment process.

After being excited by a pulse from an Nd: YAG-laser $(\lambda=1064 \mathrm{~nm})$, the cavity ring down method measures the decay of radiation leaking out of a high-finesse cavity (f 60000, reflectivity of $99.995 \%$ ).

The attenuation of the vacuum background signal by the photo detachment process due to the presence of negative hydrogen ions can be measured in a decreased decay time of the cavity. This method gains the absolute, line-of-sight integrated negative ion density in the source.

The CRDS system was recently successfully commissioned at the BATMAN test facility; the measured values of the negative ion density agree rather well with those of the optical emission spectroscopy.

\section{Laser Detachment}

The laser-detachment method [20] will be used for a relative measurement of the spatially and temporally resolved negative ion density distribution. The principle is based on the detachment of the electrons from the negative hydrogen ions by a pulsed laser beam. Then the additional electrons are detected by a Langmuir probe system, measuring in the electron-saturation region.

The probe tip is arranged to be coaxial to the laser beam. The length of the probe tip mainly determines the spatial resolution of the system. The maximal time resolution of about $60 \mathrm{~ms}$ results from the laser pulse frequency of $15 \mathrm{~Hz}$. The currents of the probe tip are usually in the order of some $\mathrm{mA}$. In order to compensate periodic changes of the plasma potential with the oscillating RF power, the laser is triggered in phase with the RF power.

\section{Local Extraction}

In order to get information about the possible ion and electron current density and the beam homogeneity, local single aperture extraction with a Faraday cup system [21] will be used. The Faraday cup system is currently in the design phase. It is planned to adapt the geometry of the present BATMAN and MANITU grid system [22] with a diameter of the plasma grid aperture of $8 \mathrm{~mm}$; the electrons are deflected by permanent magnets to specially designed electrodes similar to the deflection in actual extraction grid systems. Both electron and ion currents are measured electrically; secondary electrons are suppressed by a small negative ( 100 V) potential difference between the cup and the electron deflection electrode. A design goal is to achieve similar voltages $(8-9 \mathrm{kV})$ as in a 'real' grid system.

It is planned to have 10 to 15 of such Faraday cups distributed behind the plasma grid to achieve sufficient information of the negative ion density profile as well as on the profile of the amount of co-extracted electrons. 


\section{Results of the First Plasma Discharges}

Figure 9 shows the results of one of the first $50 \mathrm{~kW}$ RF discharges. The time trace of the RF power measurement shows an oscillation that is most probable due to a RF pick up of the signal transmission (upper part). By the matching with the two variable capacitors a value of roughly 0.8 for the cosine of the phase angle between RF current and voltage at the generator output has been achieved.

The source was operated with only one of the two RF generators, so that Plasma was ignited only in the lower two drivers. In the upper drivers no plasma was produced. In the centre of the figure the signal of the $\mathrm{H}_{\alpha}$-line for the lower drivers is shown. The $\mathrm{H}_{\alpha}$ signal, observed in the horizontal pair of drivers is almost identical, what means that the RF power is equally distributed among the drivers. The intensity in the left driver is slightly smaller than in the right driver, which can be explained by the small, at present tolerable pressure difference in the drivers shown in the lower part of figure 9 (drivers with RF).
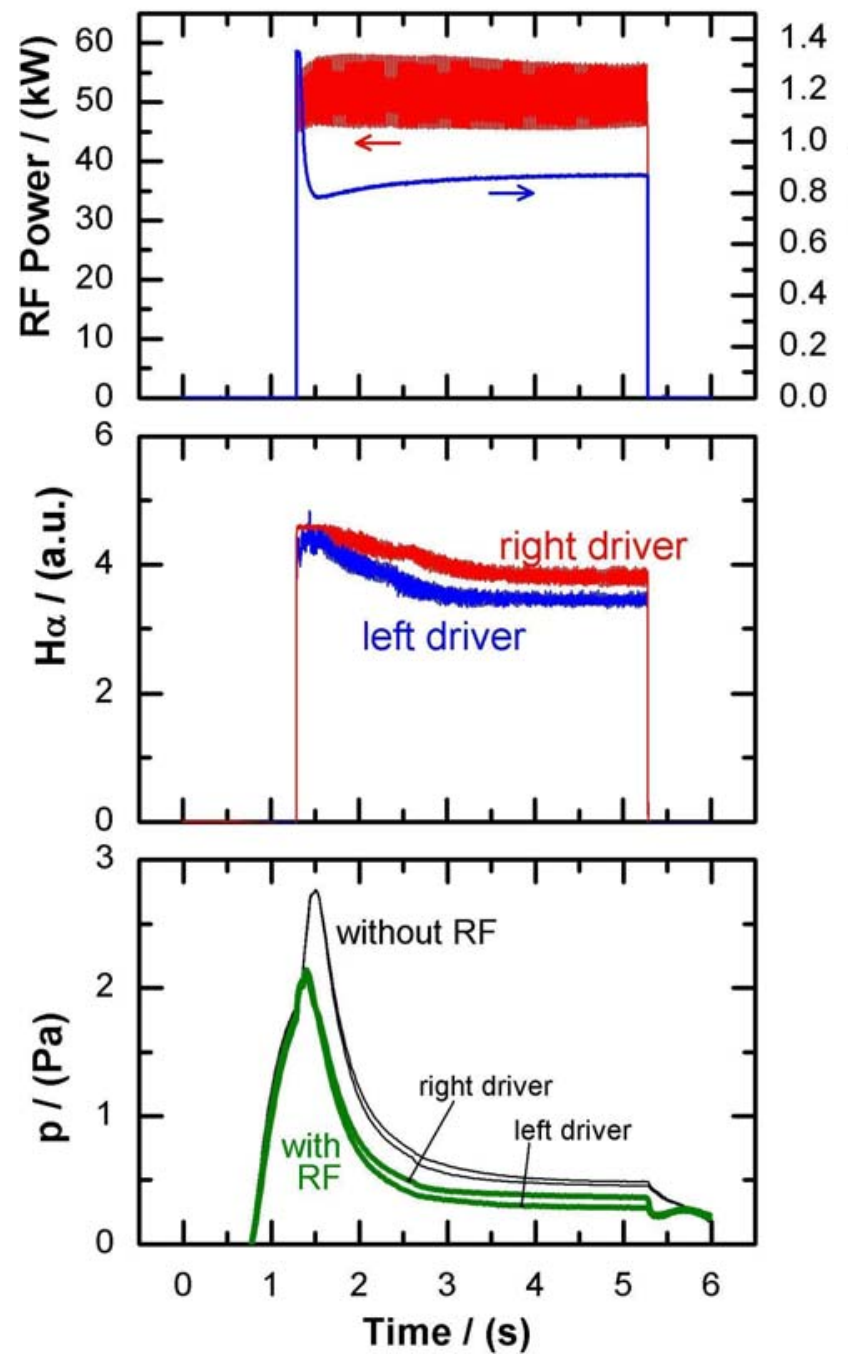

Figure 9: Upper part: time trace of the $\mathrm{RF}$ power and the cosine of the phase angle between RF current and RF on voltage. Centre: Time trace of the $\mathrm{H} \alpha$ O emission line in the drivers with RF (lower drivers). Lower part: Time traces of the pressure in the drivers with and without RF.

The time trace of the pressure in the four drivers starts with a gas puff that is needed for the ignition of the plasma discharge. After that it reaches a value of about $0.5 \mathrm{~Pa}$. The pressure during the plasma discharge in the drivers is lower than without plasma. This effect was already observed in the smaller IPP test beds [3] and can be explained by the plasma streaming out of the drivers. 


\section{Summary}

IPP Garching has developed and recently commissioned a new source at the test facility RADI, with roughly half of the size of the ITER source. This experiment is devoted to show the scaling and modular concept of the IPP RF source. The required plasma homogeneity will be measured with the implemented diagnostics accompanied by plasma modelling. The diagnostics include optical emission spectroscopy, Laser detachment, Langmuir probes, and Cavity ring down spectroscopy. Their functional capability was shown under RF conditions at the other IPP test beds. Furthermore an ITER-like RF circuit will be tested.

The source has a very flexible design. The magnetic PG filter, also foreseen for the ITER source will be examined. The source body surfaces and the Molybdenum dummy grid surface are temperature controlled.

The results of the first discharges performed, showed that two drivers can be operated in series with one generator and that good matching of the RF circuit to the RF power supply can be achieved. 


\section{Literature}

[1] ITER Technical Basis 2002 Detailed Design Document, section 5.3 (DDD5.3) (International Atomic Energy Agency)

[2] ITER Technical Basis 2002 ITER EDA Documentation Series No 24 (Plant Description Document, section 2.5.1) (International Atomic Energy Agency)

[3] E. Speth, HD. Falter, P. Franzen, U. Fantz, M. Bandyopadhyaya, et al., Nucl. Fusion 46 (2006) S220

[4] P. Franzen, H. D. Falter, E. Speth, W. Kraus, M. Bandyopadhyay, et al. Fusion Engineering and Design 74 (2004) 351

[5] W. Kraus, B. Heinemann, H. D. Falter, U. Fantz, T. Franke, et al., Fusion Engineering and Design 74 (2004) 337

[6] P. Franzen, H. Falter, B. Heinemann, Ch. Martens, U. Fantz, et al., to be published

[7] U. Fantz, H. Falter, P. Franzen, S. Christ, B. Heinemann, et al., Nuclear Fusion, 46 (2006) S 297

[8] U. Fantz, M. Bandyopadhyay, H. D. Falter, P. Franzen, B. Heinemann, et al., Fusion Engineering and Design 74 (2004) 299

[9] U. Fantz, H. Falter, P. Franzen, E. Speth, R. Hemsworth, et al. Rev. of scientific instruments 77, (2006) 03A516

[10] U. Fantz, D. Wünderlich, Submitted to New Journal of Physics

[11] S. Christ, U. Fantz. To be published

[12] M. Berger, U. Fantz. To be published

[13]E. Quandt, I. Kraemer, H.F. Döbele. Europhysics Letter, 45 (1), pp. 32-37 (1998)

[14] W. Kraus, P. Franzen, B. Heinemann, E. Speth, O. Vollmer. Fusion Engineering and Design, 56-57 (2001) 499

[15] O. Vollmer, B. Heinemann, W. Kraus, P. McNeely, R. Riedl, et al., Fusion Engineering and Design, 56-57 (2001) 465

[16] K. Ikeda, Y. Takeiri, O. Kaneko, K. Nagaoka, Y. Oka, et al., Review of Scientific Instruments 75(5), 2004, 1744

[17] N. Umeda, L.R. Grisham, T. Yamamoto, M. Kuriyama, M. Kawai, et al., Nucl. Fusion 43 (2003) 522-526

[18] R. Wilhelm, Max-Planck-Institut für Plasmaphysik, Garching, Germany. Private communication, 2003

[19] M. Bandyopadhyay, Ph.D. Thesis, Technische Universität München, 2004.

[20] M. Bacal, G.W. Hamilton, A.M. Bruneteau, H.J. Doucet, J. Taillet, Rev. Sci. Instrum. 50(6), 1979, 719

[21] P. Franzen, H. D. Falter, U. Fantz, W. Kraus, M. Berger, 24th Symposium on Fusion Technology - Warsaw, 11- 15 Sept. 2006

[22] B. Heinemann, J. Bucalossi, P. Frank, R. Riedl, A. Simonin, et al.., Proceedings of the 20th Symposium on Fusion Technology, Marseille, 1998. Association EURATOM-CEA, Saint Paul Lez Durance, 1998, p. 433 